\title{
Histologische Befunde beim sog. Pseudotumor cerebri.
}

\author{
Von \\ Dr. Stefan Rosental.
}

(Aus dem anatomischen Laboratorium der psychiatrischen Klinik in München).

Mit 5 Textfiguren und 1 Tafel.

(Eingegangen am 15. August 1911.)

Unter dem Begriff „Pseudotumor cerebri“ wurden von Nonne diejenigen Erkrankungen zusammengefaßt, bei welchen das Krankheitsbild die Veranlassung zur Annahme einer Hirngeschwulst gibt, während nach dem Ausgang der Erkrankung oder dem Sektionsbefund sich eine derartige klinische Diagnose als unberechtigt erweist. Es ist das Verdienst von Reichardt, darauf hingewiesen zu haben, daß die Erscheinungen des gesteigerten Hirndruckes durch die physikalischen Zustandsänderungen in der Hirnsubstanz hervorgerufen werden können, indem das Gehirn infolge einer festen Bindung der Gewebsflüssigkeit eine Volumensvermehrung erleidet und daraus ein Mißverhältnis zwischen der Schädelkapazität und deren Inhalt resultiert. Die anatomische Erforschung derartiger Vorgänge hat begonnen mit dem Nachweise, daß bei manchen akuten Krankheitszuständen des Nervensystems in den Gewebsstrukturen bestimmte Veränderungen auftreten, welche zu einer Anschwellung des Gehirns führen müssen (Alzheimer). Diese Volumenszunahme kann demnach verursacht werden zunächst lediglich durch die sog. ,,akute Erkrankung“ der Nervenzellen Nissls, welche der trüben Schwellung der Drüsenzellen von Virchow entsprechen dürfte und meistens bei infektiösen Erkrankungen, oft ohne schwerere Reaktionserscheinungen seitens der Neuroglia, beobachtet werden; dann kommen auch die Quellungserscheinungen an den Nervenfasern in Betracht. Viel wichtiger sind aber für das Zustandekommen der Hirnschwellung jene Umwandlungen der gliösen Stützsubstanz, welche von Alzheimer zuerst beschrieben und mit dem Namen der amöboiden Neuroglia belegt worden sind. Demgegenüber behauptet Reichardt in einem kürzlich erschienenen Sammelreferat, daß die bisherigen histologischen Befunde das klinische Bild des Hirndrucks nicht erklären könnten und er richtet an die pathologische Histologie die Aufforderung, derartige Veränderungen nachzuweisen, durch welche man die Volumensveränderungen 
des Gehirns nicht „nur erkennen, sondern auch befriedigend erklären“: könnte. Während Pötzl und Schüller unter Berücksichtigung der Alzheimerschen Ergebnisse den Begriff der Hirnschwellung auf alle diejenigen Erkrankungen auszudehnen versuchten, bei welchen es zu einer Volumensvermehrung des Zentralorgans infolge der verschiedenartigen Anomalien in Bildung und Abfluß der intrakraniellen Gewebsflüssigkeit kommt, betont Reichardt den grundsätzlichen Unterschied zwischen der Hirnschwellung in seinem Sinne und denjenigen Volumensvergrößerungen des Gesamtgehirns, welche durch eine Vermehrung der freien Flüssigkeit, sei es in den Gewebslücken des Gehirns, sei es im Hirnventrikel, zustande kommen, und bekanntlich als Hirnödem bzw. als Hydrocephalus bezeichnet werden. Es ist dabei bemerkenswert, caß auch N on ne auf Grund der klinischen Überlegungen den Unterschied zwischen dem Pseudotumor und dem Hydrocephalus betont hat, wodurch die Beziehung der Pseudotumoren zur Hirnschwellung naheliegend wurde.

Das Gebiet der idiopathischen Hirnschwellung umfaßt die $\mathbf{Z u}$ stände mit Hirndruckserscheinungen bei den folgenden Erkrankungen:

1. bei Intoxikationen, mit einer bekannten Ätiologie, also

a) bei infektiösen Erkrankungen,

b) durch exogene Gifte bedingten,

c) autotoxischen Ursprungs (z. B. bei der Urämie).

2. Manche akuten Schübe bei denjenigen Hirnerkrankungen, deren Zusammenhang mit einer Intoxikation bisher einwandsfrei nicht nachgewiesen ist, wenn auch aus manchen Ergebnissen der Stoffwechseluntersuchung wahrscheinlich erscheint, nämlirh bei der Epilepsie und bei der Katatonie.

Dann bleibt noch eben

3. eine Anzahl von Fällen, welche sich weder auf eine Intoxikation zurückführen, noch zu Epilepsie oder Katatonie einwandsfrei zählen lassen, deren nosologische Stellung unaufgeklärt ist, namentlich die Pseudotumoren.

Man darf annehmen, daß das Gemeinsame aller dieser Krankheitszustände, welche mit Erscheinungen des gesteigerten Hirndrucks einhergehen und als eine idiopathische Hirnschwellung betrachtet werden dürfen, ist die Möglichkeit das klinische Bild durch irgendwelche in der Hirnsubstanz, also lediglich in den ektodermalen Bestandteilen, liegende Veränderungen zu erklären. Dagegen müssen als eine besondere Gruppe alle diejenigen Hirnschwellungen abgetrennt werden, welche eine Komplikation verschiedenartiger intrakranieller Erkrankungsprozesse darstellen, bei welchen sich bestimmte pathologische Vorgänge vor allem an den mesodermalen Elementen, namentlich an den Hirnhäuten und in den Gefäßen abspielen, also die Hirnschwellungen bei 
der Paralyse, die bei Hirntumor, bei Hirnabsceß auftretenden, die als eine Begleiterscheinung der Encephalitis beobachteten.

Während bei den meisten derjenigen Krankheitszuständen, bei welchen eine idiopathische Hirnschwellung angenommen werden konnte, z. B. beim Status epilepticus und bei den schweren katatonischen Zuständen, abnorme Abbauvorgänge im Nervengewebe und besonders das Vorkommen der amöboiden Glia von Alzheimer nachgewiesen wurde, haben die anatomischen Untersuchungen bei den Pseudotumoren bis jetzt keine übereinstimmenden Resultate ergeben. Bei manchen Fällen, in welchen während des Lebens das Vorhandensein eines'Hirntumors angenommen wurde, ist der anatomische Befund angeblich vollkommen negativ gewesen (Nonne-S piel me yer). Dagegen fanden sich dann bei den anderen derartigen als Pseudotumor beschriebenen Fällen bestimmte intrakranielle Erkrankungsprozesse, welche die Hirnschwellung entweder als eine Komplikation hervorgerufen oder dieselbe überhaupt nur vorgetäuscht haben, indem sie allein schon die Erscheinungen des gesteigerten Hirndruckes bedingen konnten; zu dieser Kategorie lassen sich z. B. die von Weber und Schulz beschriebenen Fälle rechnen, besonders derjenige, bei welchem eine Sarkomatose der Meningen gefunden worden ist. Dann haben Finkelnburg und Eschbaum unter Hinweis auf einen entsprechenden Fall betont, daß eine basale Leptomeningitis die Erscheinungen eines Hirntumors erzeugen kann und haben deshalb vor der Annahme eines „Pseudotumors ohne anatomischen Befund" gewarnt. Es ist selbstverständlich, daß man bei derartigem Befunde die entsprechenden Krankheitsfälle überhaupt nicht weiter als Pseudotumor cerebri betrachten darf, insbesondere natürlich dann, wenn man darunter nicht nur eine klinische Verlegenheitsdiagnose, sondern eine Untergruppe der idiopathischen Hirnschwellung verstehen will. Wenn auch bei derartigen Krankheitsfällen bestimmte Veränderungen in den gliösen Strukturen, welche nach Alzhei mer als die Ursache der Volumensvermehrung des Organs betrachtet werden, sich finden, so sind dieselben auf eine gleiche Stufe zu stellen, wie die erwähnten Hirnschwellungen beim Tumor cerebri und beim Hirnabsceß, welche lediglich sekundäre Erscheinungen darstellen und keinerlei nosologische Bedeutung beanspruchen können.

Um so wichtiger sind diejenigen Fälle, welche als eigentliche Pseudotumoren betrachtet werden dürfen, bei welchen sich die pathologischen Vorgänge vorzugsweise an den ektodermalen Gewebsbestandteilen abspielen; indem nämlich diese Krankheitsbilder mit den ausgesprochenen Erscheinungen des gesteigerten Hirndrucks einhergehen, darf man die anatomischen Veränderungen bei denselben am ehesten mit der Hirnschwellung in Beziehung bringen.

Es wird deshalb hier über die Ergebnisse der anatomischen Unter- 
suchungen bei zwei Fällen berichtet, welche von Prof. Lewandowsky in Berlin klinisch beobachtet wurden.

Die eine Kranke hat folgende Krankengeschichte:

Die 28 jährige Frau G. erkrankte Mitte März 1910 mit Kopfschmerzen, hauptsächlich in der Stirn, Erbrechen, Schwindel. Sie klagte ferner über Vergeßlichkeit und Benommenheit, ferner daß sie nach rechts falle, ,grade wie wenn man be. soffen ist", endlich über Steifigkeit im Nacken und über ziemlich häufig eintretende schmerzhafte Drehungen des Kopfes nach hinten und links.

Objektiv ließen sich der cerebellare Gang mit Fallen nach rechts feststellen und die ziemlich hochgradige Nackensteifigkeit und Schmerzhaftigkeit bei Bewegung des Kopfes. Sonstige meningitische Erscheinungen fehlten. Fieber bestand nicht. Pyramidensymptome oder sonstige Hinweise auf einen circumscripten Prozeß außer den genannten fehlten. Keine Stauungspapille. Wassermann negativ. Die Diagnose wurde auf Tumor cerebri oder Meningitis serosa gestellt, die Kranke zunächst ambulant mit Jodkali behandelt. Das Krankheitsbild schwankte er heblich, zeitweise war die Kranke völlig frei, dann anfallsweise und tagelang heftigste Kopfschmerzen mit Erbrechen. Die Kranke wurde dann zur klinischen Beobachtung Mitte April ins Krankenhaus Friedrichshain aufgenommen, hier besserte sich der Zustand innerhalb einiger Tage so, daß die Kranke sich völlig beschwerdefrei fühlte und entlassen werden wollte. Nach 14Tagen am 27. April nochmalige Aufnahme wegen wieder aufgetretenen heftigen Beschwerden. Jetzt wurde eine Neuritis optica festgestellt. Lumbaldruck $450 \mathrm{~mm}$ Wasser. Punktat o. B. Der cerebellare Gang war wieder deutlich, sonst kein Befund außer der auch schon früher erwähnten Nackensteifigkeit und Schwerbeweglichkeit des Kopfes. Subjektiv dauernd heftige Kopf- und vor allem Nackenschmerzen. Die Kranke saß mit nach hinten gezogenem Kopf aufrecht im Bett, laut jammernd über die Schmerzen, die aus dem Nacken und dem Rücken zeitweise auch in die Extremitäten ausstrahlten. Puls 56-64. Temperatur normal.

Die Zeichen des gesteigerten Hirndrucks waren zweifellos. Soweit lokalisierbare Symptome da waren, wiesen sie auf die hintere Schädelgrube. Es waren das der cerebellare Gang, die heftigen Nackenschmerzen, die Nackensteifigkeit. Es wurde an einen Cysticercus des vierten Ventrikels gedacht, bei dem ähnliche Bilder gesehen worden sind. Dafür konnte auch der auffallende Wechsel in der Intensität der Symptome sprechen. Die Möglichkeit einer Meningitis serosa bzw. Hydrocephalus wurde daneben betont; gegen einen eigentlichen entzündlichen Prozeß an den Meningen sprach das Fehlen eines pathologischen Zellbefundes im Liquor. Bei der Prognose wurde vor allem die Möglichkeit eines plötzlichen Exitus betont. 3 Fälle mit ähnlichen Symptomenkomplexen, die L. gesehen hat, und die sich als Cysticercus oder Tumor des vierten Ventrikels herausstellten, sind alle plötzlich zugrunde gegangen. Die Indikation zu einer Trepanation schieu jedenfalls gegeben. Die Kranke bat auch selbst um eine Operation, da die Schmerzen nicht auszuhalten seien. In den letrten Tagen schrie sie stundenlang.

Es wurde (3. Mai) eine Trepanation von Fünfmarkstückgröße über dem Kleinhirn gemacht, (dir. Arzt Dr. W. Braun), die Dura gespalten, die Wunde dann tamponiert und geschlossen, in der Absicht, nach einigen Tagen sie wieder zu öfnen und nachzusehen, ob sich ein erreichbarer pathologischer Befund nachweisen lassen würde.

Als die Kranke, die die Operation gut überstanden zu haben schien, nach dem Verbande ins Bett gelegt wurde, trat plötzlich Atemstillstand ein bei gut fühlbarem Puls. Trotz zweistündiger künstlicher Atmung - solange blieb der Puls fühlbar - Exitus. 
Die Autopsie (20 Stunden p. m.) ergab nichts als einen geringen Hydrocephalus. Auch der vierte Ventrikel war etwas erweitert, aber durchaus nicht verhältnismäßig mehr als die Großhirnventrikel. Eine Erklärung des schweren Krankheitsbildes war aus der Sektion nicht zu erhalten.

Der andere Fall erkrankte mit Kopfschmerzen, wurde benommen mit unklaren Symptomen, vorzugsweise cerebellaren Charakters, eingeliefert; auf einem Auge bestand eine Neuritis optica, in einigen Tagen trat Erblindung (mit nachfolgender Atrophie) ein. Darauf hatten sich die allgemeinen Symptome fast ganz zurückgebildet, nach einigen Wochen darn erneute schwere Benommenheit, es folgte eine in wenigen Tagen vollständige Erblindung des zweiten Auges unter Neur. opt. Nach einer dekompressiven Trepanation, welche einen Hirnprolaps zur Folge hatte, nur vorübergehender Erfolg, dann trat Exitus ein. Bei diesem Falle ergab die Obduktion (24 St. p. m.) ein makroskopisch völlig negatives Resultat, speziell fehlte auch Hydrocephalus.

Die beiden Gehirne wurden mit der Diagnose Pseudotumor cerebri an Prof. Alzheimer übersandt.

Die mikroskopische Untersuchung hat in beiden Fällen Veränderungen, welche bis zu einem gewissen Grade übereinstimmen, nachgewiesen; zuerst wird deshalb der gemeinsame Befund geschildert.

Bei der Färbung mit Hämatoxylin und mit den basischen Anilinfarbstoffen erweist sich die Rindenarchitektonik als erhalten. Die Ganglienzellen der Rinde zeigen an manchen Stellen das Bild einer ,akuten Zellerkrankung" Nissls, meistens aber besteht eine ,wabige" Anordnung der färbbaren Substanzen des Plasmas, wobei die Protoplasmafortsätze öfters angeschwollen sind, der Zellkern aber keine bedeutendere Veränderung darbietet. An den anderen sieht man die der „,schweren Erkrankung“" entsprechenden Formen. (Taf.IX, Fig. 1, Abb.b.) Das letztere Verhalten läßt sich nur teilweise erklären durch die mit Scharlach III nach Herxheimer gefärbten Präparate, welche eine starke Anhäufung der fettartigen Körnchen in den Protoplasmamaschen der Ganglienzellen zeigt. Während aber die lipoiden Substanzen meistens an der Basis der Ganglienzellen angehäuft sind, sieht man hier die Vakuolisation im ganzen Zelleib, auch in den Protoplasmafortsätzen, ziemlich gleichmäßig verteilt, so daß man diese Bilder als eine Abart der Verflüssigungsvorgänge der Nervenzellen, namentlich als den $\mathrm{Be}-$ ginn einer ,,schweren Zellerkrankung" zu betrachten berechtigt ist. Die Gliazellen der Rinde sind relativ weniger, dagegen die Gefäße stark verfettet, was ebenfalls schon an den mit basischem Anilinfarbstoff gefärbten Präparaten in Form der sog. ,grünlichen“ Abbaustoffe Alzheimers zum Vorschein kommt. Diese Substanzen finden wir auch in den Meningen abgelagert, welche daneben geringe Mengen von Blutpigment enthalten. 
Diese degenerativen Veränderungen beweisen nur, daß sich in der Rinde abnorme destruktive Vorgänge abgespielt haben. Eine weitere Aufklärung darüber gibt uns das Verhalten der Neuroglia. Wenn wir im Thioninpräparat die Gliazellen der Rinde betrachten (Taf. IX, Fig. 1), so sieht man, daß viele Trabantzellen vollkommen normal aussehen (ngl.); sie haben ihren meistens ovalen Kern, welcher viel achromatisches Karyoplasma, darüber ein breitmaschiges, zartes Chromatingerüst besitzt, und einen metachromatischen, öfters randständigen Nucleolus enthält. Dem gegenüber sind manche Trabantzellkerne (pak.) bedeutend kleiner, vollkommen rund; der gesamte Kerninhalt färbt sich stark mit basischen Anilinfarbstoffen und ist soweit homogen, daß man weder die Chromatinsubstanzen noch den Nucleolus in dem gleichmäßig dunklen Karyoplasma sieht; die Kernwand ist verdickt und manchmal von Kerninhalt durch einen dünnen, lichtbrechenden Ring abgehoben. Um den Kern sieht man meistens einen hellen leeren Hof, welcher öfters durch radiär angeordnete Plasmakörnchen überbrückt wird. Besonders deutlich tritt die Homogenität derartiger Gliakerne und die Plasmastrukturen in den mit der Mannschen Farblösung nach der Alzheimer schen Methode V hergestellten Präparaten hervor (Taf. IX, Fig. 2b) ${ }^{\mathbf{1}}$ ). Diese Gliaformen sind insofern von Bedeutung, als auf Grund der verschiedenen experimentellen Befunde wir die Utberzeugung gewonnen haben, daß es sich hier um Vorstufen der amöboiden Gliazelle bzw. um präamöboide Kernformen handelt.

Während die geschilderten Veränderungen in der Rinde bei den beiden Fällen ziemlich gleichartig sind, so verhält es sich etwas verschieden in den tiefen Gehirnteilen, welche jetzt gesondert betrachtet werden. Beim Fall I sehen wir in der Markleiste viele kleine präamöboide Kerne und eine anscheinliche Vermehrung der faserbildenden Gliazellen, welche einerseits an ihrer Peripherie zahlreiche Vakuolen mit fettartigen Substanzen enthalten, anderseits eine pyknotische Verkleinerung des Kerns und eine Homogenisation des Plasmas zeigen, was ebenfalls als der Beginn einer amöboiden Umwandlung zu betrachten ist. Daneben treten im Mark zahlreiche ausgebildete amöboide Gliazellen auf : einerseits die Form mit dem homogenen Plasma und lipoiden Vakuolen, welche im S.-Fuchsin-Lichtgrünpräparat die Altmannschen Granula enthält, andererseits auch manche mit feinkörnigen Methylblaugranula beladenen Elemente.

Es lassen sich hier auch manche Ubergangsformen zwischen den

1) Diese Methode wurde bei allen folgenden Bildern angewandt; da das Material in Formol fixiert worden ist, so wurden die Gefrierschnitte zuerst auf 3-5 Tage bei Temperatur $37,5^{\circ}$ in die Weigertsche Gliabeize gelegt, davon kamen einige Schnitte zur Darstellung der lipoiden Einschlüsse auf 24 Stunden in die Flemmingsche Lösung, wonach das eigentliche Alzheimersche Verfahren folgte. 
präamöboiden Kernen und den typischen amöboiden Gliazellen nachweisen (Taf. IX, Fig. 4 u f). Man sieht an manchen Stellen, daß die Plasmabrücken, welche vom präamöboiden Gliakern radiär ausgehen, sich an der Peripherie der Zelle verdicken und zusammenfließen; in dieser Weise entstehen aus den Hohlräumen des Gliareticulums, welche dem Gliakern anliegen, die von Plasma umgebenen hydropischen Vakuolen.

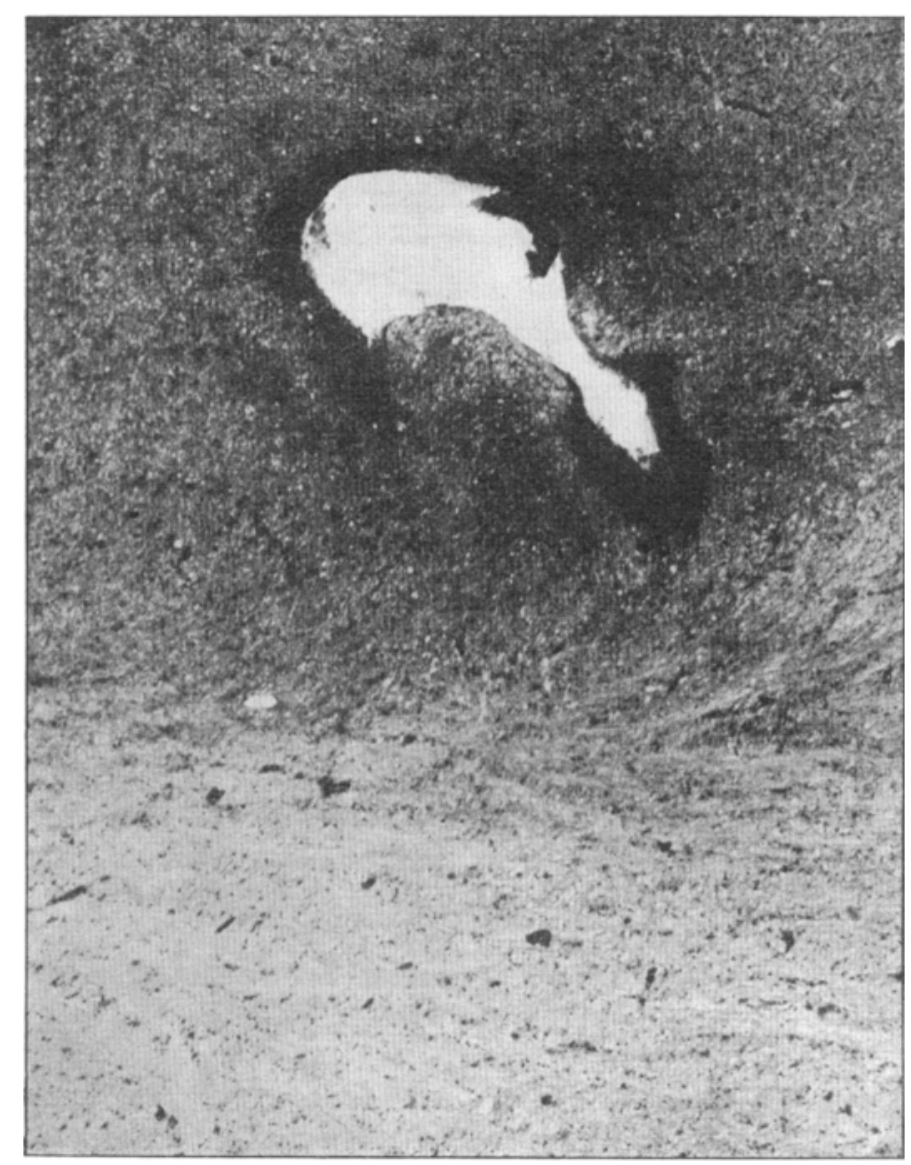

Fig. 1. Pseudotumor I. Die Ventrikelwand mit einem Divertikel.

Derartige Veränderungen sind aber noch viel ausgesprochener, wenn man weiter in die Tiefe geht und sich dem Ventrikel nähert. Bei der Betrachtung der Wand des vierten oder des Seitenventrikels (Textfig. 1) kann man am Ependymepithel bei einer starken Vergrößerung vielleicht eine Anschwellung der Epithelzellen, sonst nichts Charakteristisches feststellen. Das subepitheliale innere Gliafaser- 
geflecht erscheint verdickt, was aber mit einer Vorsicht zu verwerten ist, weil nach den klassischen Schilderungen von Weigert die ependymäre Schicht eine ungemein wechselnde Dicke haben soll; auf der Oberfläche sind die Gliafasern wenig gefärbt, dafür sieht man aber bei

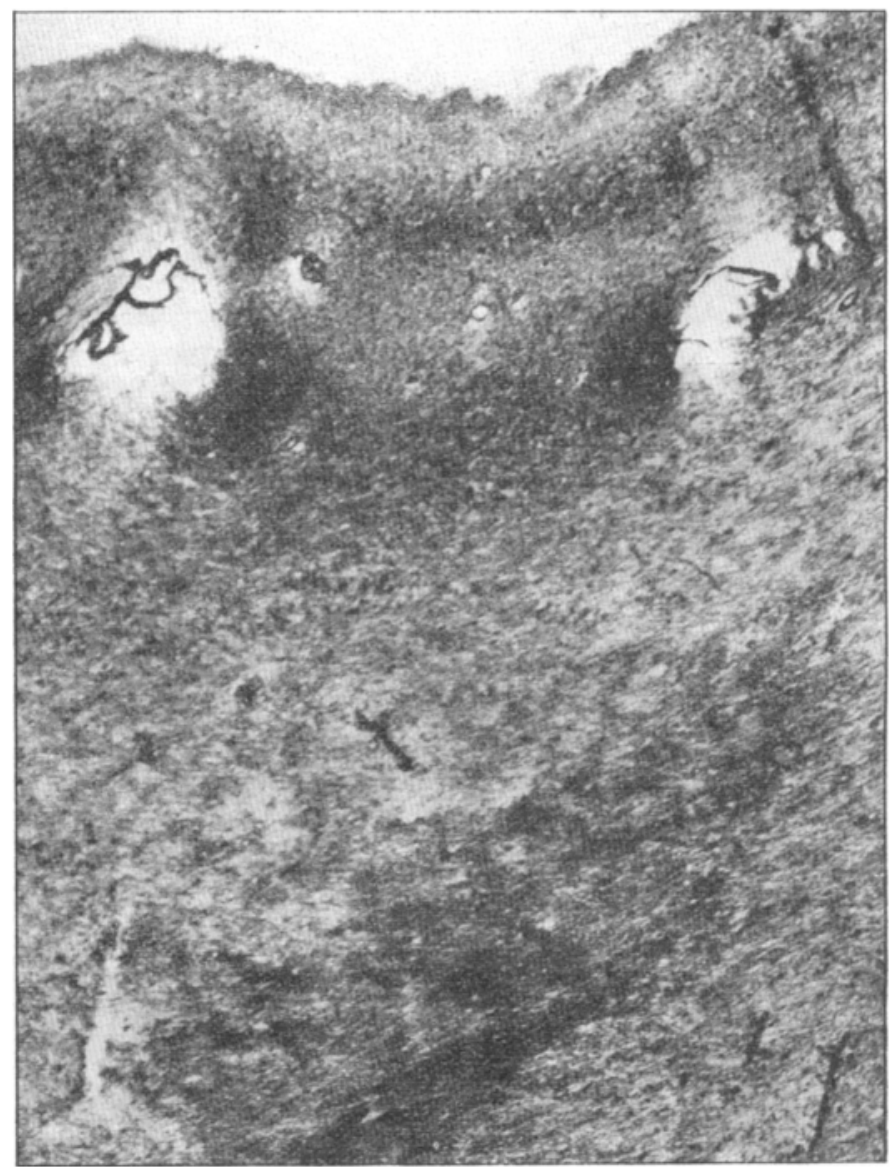

Fig. 2. Pseudotumor 1. Die Vermehrung der Gliafasern in den tiefen Schichten der Ventrikelwand.

einer starken Vergrößerung zahlreiche mit Methylblau gefärbte körnige Massen.

Die darunterliegenden subependymalen Gliamaschen zeichnen sich dagegen durch eine ausgesprochene Vermehrung der Gliafasern (Textfig. 2), welche besonders in die Gefäße ovale Narben (Textfig. 3) bilden, aus; an manchen Stellen ließen sich innerhalb derartiger Verödungs- 
bezirke die mit Fett beladenen anscheinend gliogenen Körnchenzellen nachweisen.

In der folgenden ,tiefen“ Zone des paraventrikulären Gligaeflechtes, welche normalerweise aus lockeren, meistens horizontalen Gliazügen besteht, treten massenhaft große amöboide Gliazellen auf; das mächtig angeschwollene Plasma dieser Elemente, welche anscheinend aus den gewucherten Monstregliazellen hervorgegangen sind, ist meistens homogen und enthält lipoide Vakuolen (Taf. IX, Fig. 4); viele dazwischenliegende Gliakerne entsprechen dem präamöboiden Typus. Wenn man derartige Bilder mit den normalerweise vorkommenden Gliaformen vergleicht, so wird ersichtlich, daß diese amöboide Umwandlung der Glia eine

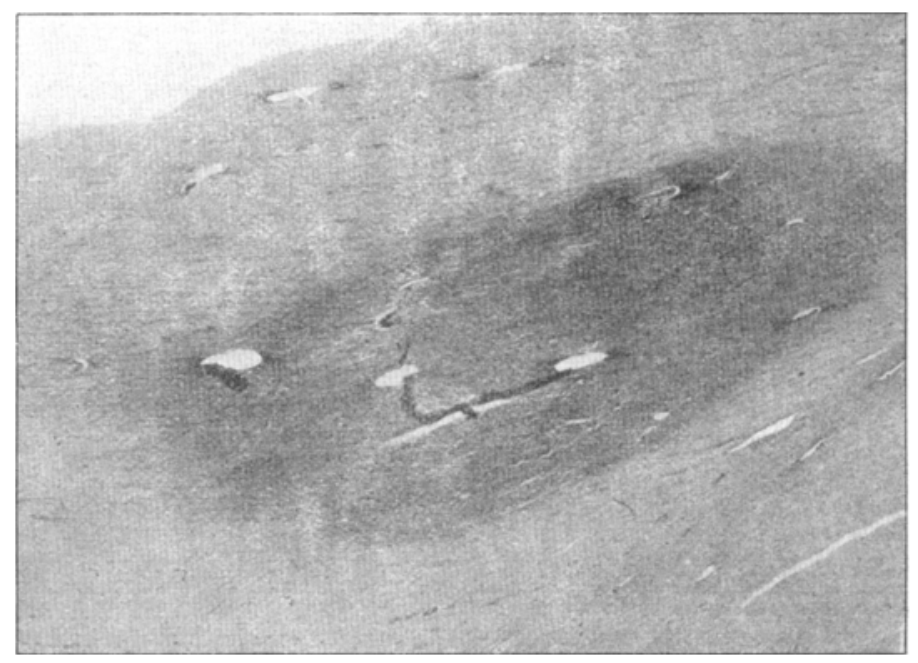

Fig. 3. Eine gliöse Narbe in den tiefen Schichten des subependymalen Gliafasergeflechtes.

Volumenszunahme des betreffenden Bezirkes hervorrufen muß; und derartige Veränderungen sind hier nicht nur in dem Markmantel vorhanden, sondern sie erstrecken sich auch weit in die tiefen Schichten der Ventrikelwandungen, also in das Gebiet der basalen Ganglien und in die Oblongata.

Was das Verhalten der mesodermalen Elemente in diesem Falle anbetrifft, so stellt es sich bei einer genauen Betrachtung heraus, daß die Leptomeningen an manchen Stellen leicht mit lymphocytären Elementen infiltriert sind; daneben sieht man auch manche mit Blutpigmenten beladene Abräumzellen. Es ist auffallend, daß alle Blutgefäße enorm stark dilatiert und mit Blut gefüllt sind. An wenigen Stellen finden sich in den adventitiellen Lymphräumen der Gefäße 
ebenfalls einige Infiltrationszellen und hämosiderinhaltige Körnchenzellen. Derartige unbedeutende Veränderungen am Mesoderm lassen sich aber keineswegs als eine Ursache der Veränderungen am Nervenparenchym auffassen; es wurden nämlich von Reichardt und dann von Redlich auch bei den Hirntumoren in der Rinde disseminierte kapilläre Blutungen gefunden. Vielmehr könnte man also diese unbedeutenden und wenig charakteristischen Veränderungen in Verbindung mit dem gesteigerten Hirndruck und der dabei bestehenden Hyperämie insofern in einen Zusammenhang bringen, als vielleicht durch die kapillären Blutungen die reaktiven Vorgänge am Mesoderm

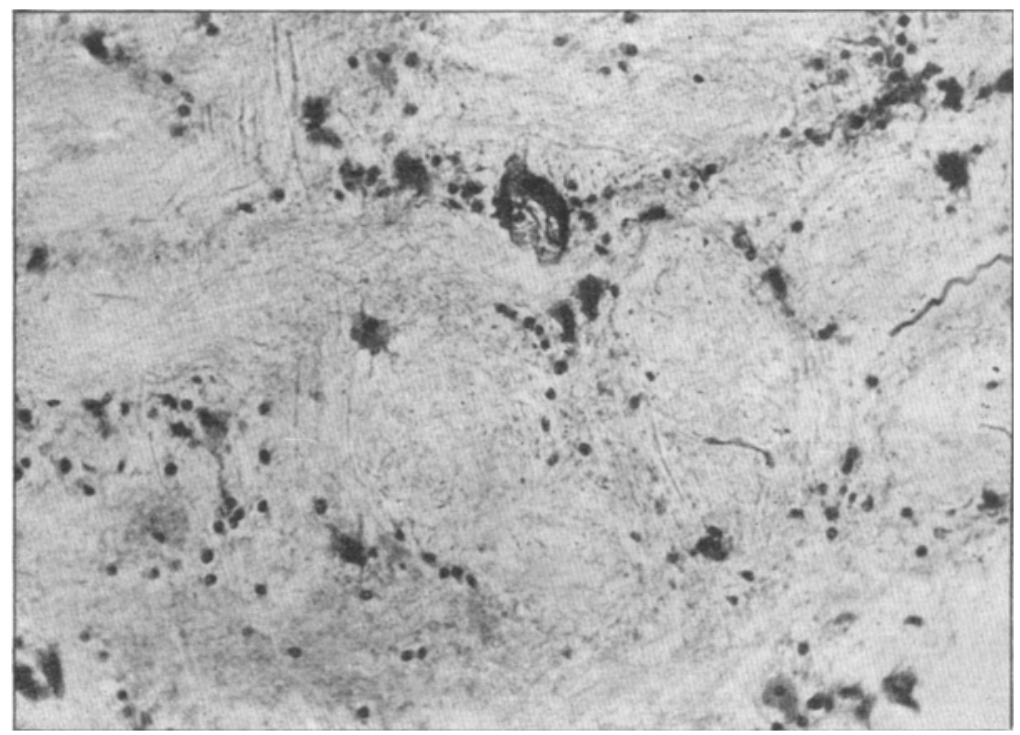

Fig. 4. Pseudotumor I. Amöboide Gliazellen in den basalen Faserzïgen.

zwecks der Entfernung des Extravasates hervorgerufen worden sind. Andererseits aber erinnern diese Befunde in mancher Hinsicht auch an einen von Pötzl und Schüller beschriebenen Fall, bei welchem die Entstehung einer Hirnschwellung durch eine luetische Infiltration der Pia und des Ependyms begünstigt wurde, und infolge der toxischen Hyperämie dabei capilläre Blutungen entstanden sind, so daß an manchen Stellen das Bild einer hämorrhagischen Encephalitis zustande gekommen ist. Nach dem Ergebnis der Serumuntersuchung kommt die Lues bei unserem Fall nicht in Betracht; auch die erwähnten Gefäßveränderungen geben keine Anhaltspunkte für eine derartige Annahme. Als das charakteristische Merkmal dieses Falles ist demnach eine schwere Umwandlung der Neuroglia besonders in den 
tiefen Hirnteilen zu betrachten, obwohl mit Hinsicht auf die Beteiligung der Meningen und der Gefäße wir in Erwägung ziehen mußten, ob man diesen Fall von Pseudotumor zu der Gruppe der idiopathischen Hirnschwellung rechnen darf.

Es ist dabei erwähnenswert, daß in der Umgebung von zwei Hirntumoren, welche gemeinsam mit F. H. Lew y untersucht wurden, sich vollkommen ähnliche Veränderungen der Neuroglia, wie beim geschilderten Pseudotumor in der Umgebung des Ventrikels, sich gefunden haben: also einerseits eine starke Wucherung der faserigen Glia, welche zur Bildung der vielverzweigten und lipoide Abbaustoffe enthaltenden Monstregliazellen führte, und andererseits eine amöboide Umwandlung derselben mit Plasmahomogenisation und Kernschrumpfung.

Ebenso vorsichtig muß bei der Rubrizierung des zweiten Pseudotumors verfahren werden, und zwar aus dem Grunde, weil das gesamte anatomische Bild hier schwächer ausgedrückt ist und besonders die Veränderungen an den Gefäßen geringfügiger sind. Nur an manchen Stellen der Markleiste läßt sich vielleicht eine leichte Andeutung der Proliferation an den Gefäßendothelien annehmen, und man findet ganz vereinzelt einige Plasmazellen, was bekanntlich weithin nicht ausreicht, um irgendwelchen bestimmten pathologischen Prozeß anzunehmen. Es ist im Gegenteil zu betonen, daß auch ähnliche, leichte proliferative Erscheinungen an den Gefäßwänden Redlich in der Rinde bei Hirntumoren beobachtet hat.

Die Veränderungen der protoplasmatischen Glia besitzen denselben Charakter wie im ersten Falle, sind aber weniger ausgesprochen; so finden wir in der Marksubstanz (Taf. IX, Fig. 2) neben den präamöboiden Kernen $(b)$ auch ziemlich viele normale Gliaelemente $(a)$; aber in der Gegend des Ventrikels, besonders in der dritten lockeren Zone der Ventrikelwand, erscheinen die typischen amöboiden Gliazellen (Taf. IX, Fig. 5), welche neben den lipoiden Einschlüssen auch viele hydropische Vakuolen enthalten. Daneben findet man zahlreiche Ubergangsformen der faserbildenden Gliazellen (Taf. IX, Fig. 3), welche aus der Auflösung der Gliafasern $(c-e)$ und der Homogenisation des Plasmas $(f-i)$ oder aus einer Schrumpfung des Kerns ihre amöboide Umwandlung erkennen lassen. An einigen Stellen der Ventrikelwand sieht man noch die höckerigen Ependymerhebungen (Textfig. 5), sonst aber läßt sich im Gegensatz zum ersten Falle keine Vermehrung der faserigen Glia feststellen.

Das Wesentliche der anatomischen Befunde in beiden Fällen besteht demnach in einer amöboiden Umwandlung der gliösen Strukturen, welche mit einer besonderen Ausprägung um die Wandungen der Hirnventrikel aufgetreten ist.

Der eine Fall ist auch insofern bermerkenswert, als bei der Sektion ein angeblich unbedeutender Hydrocephalus gefunden wurde. Es wurde 
nämlich von Apelt ein Fall von Pseudotumor beschrieben, bei welchem die physikalische Untersuchung neben dem Hydrocephalus auch eine Volumenzunahme des Gehirns resp. die Hirnschwellung ergeben hat. Dementsprechend hat Reichardt die Meinung vertreten, daß die Bildung eines Hydrocephalus die Folge einer primären Störung in der Hirnsubstanz sein könnte. Auch von Lewandowsky wurde kürzlich diese Möglichkeit in Erwägung gezogen. Infolgedessen haben wir einen als Hydrocephalus idiopathicus bei der Obduktion diagnostizierten Fall histologisch untersucht, wobei sich, übrigens beim Fehlen jeder Veränderung an den Hirnhäuten und Gefäßen, vollkommen

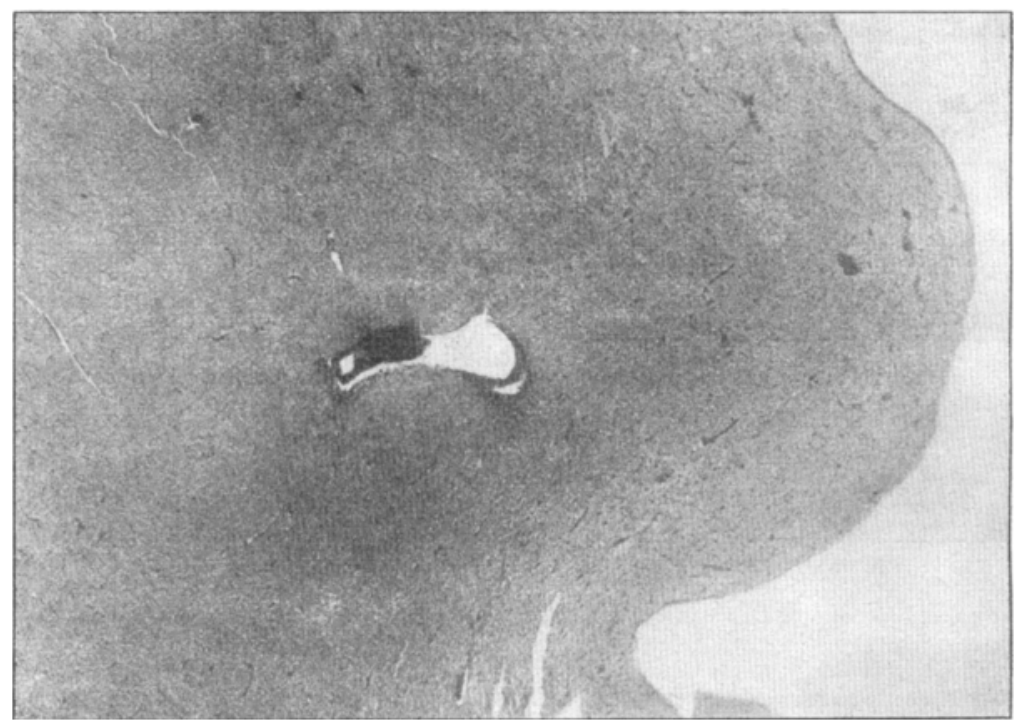

Fig. 5. Pseudotumor II. Ventrikelwand.

ähnliche Umwandlungen der Neuroglia an der Ventrikelwand, wie bei dem zweiten Pseudotumor, vielleicht noch etwas weniger ausgesprochen, fanden.

Es ist dabei bemerkenswert, daß bei allen diesen Fällen die Epithelien des Plexus chorioideus äußerst stark mit den Altmannschen Granula beladen waren; besonders ausgeprägt ist das beim letzteren Fall von Hydrocephalus idiopathicus gewesen.

Diese Befunde befinden sich in einer gewissen Ubereinstimmung mit der von Pötzl und Schüller vertretenen Ansicht, daß beim $\mathrm{Zu}$ standekommen der Hirnschwellung abnorme Verhältnisse in der Bildung und im Abfluß der intrakraniellen Gewebsflüssigkeit mitwirken sollen. Eine derartige Annahme scheint für eine Anzahl der Fälle wirk- 
lich zutreffend zu sein, ohne daß man selbe für die Erklärung aller Hirnschwellungszustände ohne weiteres heranziehen dürfte.

Was die Beziehungen derartiger Erkrankungen zur Katatonie anbetrifft, so hat schon Reichardt betont, daß manche Pseudotumoren sich durch das Auftreten der psychomotorischen Störungen auszeichnen. Wenn alle diejenigen als Pseudotumor diagnostizierten Krankheitsbilder, bei welchen irgendwelche intrakranielle Erkrankungen die idiopathische Hirnschwellung nur vorgetäuscht haben, vollkommen auf die Seite gestellt würden, so wäre wahrscheinlich diese symptomatologische Eigentümlichkeit noch auffallender und man müßte wohl Reichardt zustimmen, daß viele von den wirklichen Pseudotumoren zur Katatonie hinzugerechnet werden dürfen. Es ist in dieser Hinsicht sehr charakteristisch ein von $\mathrm{Knauer}$ beobachteter Fall von Pseudotumor, bei welchem nach einer katatonischen Erregung ein Status epilepticus mit hemiparetischen Erscheinungen aufgetreten ist. Diese Beziehungen zwischen Pseudotumoren und der Katatonie haben uns veranlaßt, die Lokalisation der anatomischen Veränderungen bei den akuten katatonischen Zuständen, welche sich nach den Forschungen von Alzheimer und Eisath ebenfalls' durch das Auftreten der amöboiden Neuroglia auszeichnen, zu berücksichtigen. Besonders wichtig sind die plötzlichen Todesfälle bei der Katatonie, welche auch von Reichardt als das Beispiel eines Hirntodes infolge der Hirnschwellung angeführt werden. Bekanntlich hat Alzheimer in einem derartigen Falle die amöboiden Gliazellen nachgewiesen. Es wurde uns kürzlich von Herrn Dr. Bonfiglio das Gehirn eines in ähnlicher Weise plötzlich verstorbenen Katatonikers überlassen, in welchem wir die gleichen Veränderungen der Neuroglia bezüglich der Intensität und Ausdehnung wie beim zweiten Pseudotumor fanden: also präamöboide Gliakerne in der Rinde und in der Marksubstanz, während in der Nähe der Ventrikelwand die amöboiden Gliazellen mit homogenem Plasma vorhanden waren.

Gegen die Verwertung dieser Befunde bei den Katatonikern, welche zwar perakut zugrunde gegangen sind, aber keine deutlichen Erscheinungen des gesteigerten Hirndrucks dargeboten haben, könnte eingewendet werden, wie es Reichardt in seinem Referat gegen den Alzhei merschen Fall getan hat, daß die Hirnschwellung physikalisch nicht festgestellt worden ist. Es fragt sich aber, ob aus jeder Hirnschwellung eine Volumenszunahme des gesamten Gehirns resultieren muß. Wie es Reichardt selbst betont hat, bleiben öfters Schwellungen auf bestimmte Bezirke beschränkt, und mit besonderer Vorliebe lokalisieren sich dieselben in der Gegend der basalen Ganglien. Es kann demnach vorkommen, daß z. B. ein Paralytiker, dessen Rinde verödet, also infolge des Ausfalls der nervösen Elemente eine Volumensvermin- 
derung erlitten hat, eine interkurrente Schwellung in den lebenswichtigen Zentren bekommt und an derselben zugrunde geht, oder z. B. bei einem Katatoniker entsteht die letale Hirnschwellung auf dem Boden des vierten Ventrikels und der Kranke stirbt am Vagustod; dann gibt es, wie Nonne hingewiesen hat, eine Kategorie der Pseudotumoren, bei welchen der lokale Hirndruck lediglich cerebellare Symptome erzeugt. Wenn man aus den Angaben Reichardts Schlüsse zieht, so braucht bei vielen derartigen Fällen das Gesamtvolumen des Gehirns keine Vergrößerung zu erleiden; während die physikalische Untersuchung heutzutage auch über keine Methoden verfügt, welche die Schwellung der einzelnen Hirnbezirke feststellen können, kann die histologische Untersuchung verschiedener Teile uns die Klärung über die Ursachen des lokalen Hirndrucks verschaffen.

Wenn wir unsere Befunde zusammenfassen sollen, so ergibt sich, daß ebenso bei einigen mit chronischen Erscheinungen des gesteigerten Hirndrucks einhergehenden Erkrankungen, wie bei manchen akuten Zuständen, welche als das Beispiel einer Hirnschwellung angeführt werden, sich die gleichartigen Veränderungen der Glia, welche besonders in der Ventrikelwand, also in basalen Ganglien und in Balken, ausgesprochen waren, fanden. Indem diese Gegend bei Zustandekommen der psychomotorischen Leistungen beteiligt ist, so könnte man denken, diese eigentümliche Lokalisation mit den katatonisehen Zustandsbildern, worauf schon Kleist hingewiesen hat, in Zusammenhang zu bringen. Ebenso wie z. B. Bonhoeffer die katatonischen Zustandsbilder bei den symptomatischen Psychosen als einen klinischen Reaktionstypus aufgefaßt hat, so wäre naheliegend, die paraventrikuläre Schwellung als einen histologischen Reaktionstypus des Gehirns und die Grundlage mancher klinischer Symptome zu betrachten. Eine derartige bestimmte Deutung unserer Befunde erscheint uns aber verfrüht; und zwar ganz abgesehen von der vielumstrittenen Frage über das Wesen der psychomotorischen Störungen schon aus dem Grunde, weil die Entstehungsbedingungen der amöboiden Veränderung der Glia und die Bedeutung verschiedener Abarten derselben noch nicht vollständig aufgeklärt ist. In seiner grundlegenden Arbeit hat Alzheimer hingewiesen, daß die die fuchsinophilen Granula und die lipoiden Einschlüsse enthaltenden Gliaformen, welche sich auch bei den Pseudotumoren fanden, mit den pathologischen Abbauvorgängen, welche durch bestimmte Krankheitsprozesse bedingt werden, in Zusammenhang zu bringen sind. Andererseits sollen die mit Methylblaugranula beladenen Zellen und die entsprechenden pathologischen Produkte bei den besonders stürmisch verlaufenden Vorgängen entstehen.

Dementsprechend ist es uns gelungen, durch verschiedene experimentelle Intoxikationen eine Schwellung der gliösen Strukturen und 
die entsprechenden destruktiven Veränderungen der nervösen Elemente zu erzeugen, worüber demnächst ausführlicher berichtet wird. Es hat sich aber dabei herausgestellt, daß im Nervensystem normaler Tiere, welches keinerlei Veränderungen direkt nach dem Tode dargeboten hat, jedenfalls wenn es unter Zimmertemperatur gehalten wird, schon innerhalb 12 Stunden nach dem Tode die Gliazellen postmortale Veränderungen erleiden können, welche mit gewissen Formen der amöboiden Gliazellen, namentlich mit den die pyknotischen Kerne und Methylblaugranula enthaltenden Elementen, vollkommen übereinstimmen, sowie daß auch eine ausgesprochene Quellung der Achsenzylinder und Verflüssigung der Nervenzellen entstehen können, was mit der Angabe von Eisath über die angebliche Widerstandsfähigkeit der gliösen Strukturen und besonders der Zellkerne gegenüber der Leichenveränderung sich in einem Widerspruch befindet. Diese postmortalen Schwellungszustände, welche anscheinend auch eine Volumenszunahme des Organs erzeugen, und vielleicht durch eine Bindung der Gewebsflüssigkeit infolge der abnormen Säuerung der Leiche bedingt werden, sind insofern von einer großen biologischen Bedeutung, als sie beweisen, daß die amöboide Umwandlung der Glia auf physikalisch-chemischen Vorgängen in der Hirnsubstanz beruht. Andererseits aber erschwert das bedeutend die Klärung mancher Sektionsbefunde überhaupt und besonders bei den mit Erscheinungen des gesteigerten Hirndrucks einhergehenden Erkrankungen. Wenn z. B. ein Kranker nach einem akuten katatonischen Zustand an einer septischen Erkrankung zugrunde gegangen ist und dazu noch nicht sofort nach dem Tode seziert wurde, so wird es vorläufig viel schwieriger, den Befund der amöboiden Gliazellen in klinischer Richtung zu deuten. Wenn insbesondere während derartiger Erkrankungen oder sogar im agonalen Zustand die Erscheinungen des gesteigerten Hirndrucks bestanden haben und man bei der anatomischen Untersuchung die mit Methylblaugranula beladenen amöboiden Gliazellen in der Ventrikelwand findet, so läßt sich auf Grund eines derartigen Zellbildes nicht sicher entscheiden, ob derartige Veränderungen während des Lebens bestanden haben und inwiefern vielleicht ein intravitaler Hydrocephalus oder Ödem eine Quellung der gliösen Strukturen in der Agone oder überhaupt erst nach dem Tode begünstigen konnte. Es muß das anatomische Gesamtbild, namentlich das Verhalten der Abbaustoffe, und die Beziehung desselben zu dem klinischen Bilde berücksichtigt werden; vor allem aber diejenigen histologischen Methoden, welche über die funktionellen Zustände der Zellen urteilen lassen, namentlich der von Alzheimer in die Neurohistologie eingeführten Färbungen der Altmannschen Granula. Die vergleichenden Untersuchungen über die amöboide Umwandlung der Neuroglia und deren Beziehung zu den 
postmortalen Gliaveränderungen werden in den Nissl - Alzheimerschen Arbeiten erscheinen. Viel verhängnisvoller sind jedenfalls die postmortalen Schwellungsvorgänge für die Untersuchung des Gehirns mittels der Wage, und besonders bedenklich erscheint die auf Grund der postmortalen Wägungen von Reichardt postulierte scharfe Trennung der Hirnschwellung als einer intravitalen festen Bindung der Gewebsflüssigkeit von einer Ansammlung der freien Organlymphe, welche dem Hirnödem und dem Hydrocephalus zugrunde liegt.

\section{Literaturverzeichnis.}

1. Alzheimer, Über den Abbau des Nervengewebes. Allg. Zeitschr. f. Psych. 63, 568. 1906.

2. - Beiträge zur Kenntnis der pathologischen Neuroglia. Nissl-Alzheimers Arbeiten 3, 401. 1911.

3. A pelt, Der Wert der Schädelkapazitätsmessungen und der vergleichenden Hirngewichtsbestimmungen. Deutsche Zeitschr. f. Nervenheilk. 35, 35. 1908.

4. Bonhöffer, Die symptomatischen Psychosen. Wien 1910.

5. Eisath, Über normale und pathologische Histologie der menschlichen Neuroglia. Monatsschr. f. Psych. 20. 1906.

6. Finkelburg und Eschbaum, Zur Kenntnis des sog. Pseudotumor cerebri mit anatomischem Befund. Deutsche Zeitschr. f. Nervenheilk. 38, 35. 1910.

7. Kleist, Die psychomotorischen Störungen bei Geisteskranken. Leipzig 1908.

8. K nauer, Über Stoffwechselstörungen in einem Falle von Pseudotumor cerebri. Centralbl. f. Nervenheilk. u. Psych. 30, 669. 1907. (Ref.)

9. Lewandowsky, Erfolgreiche Trepanationen ohne Befund. Therap. Monatshefte 1911.

10. Pötzl, Zur Frage der Hirnschwellung und ihrer Beziehung zur Katatonie. Jahrb. f. Psych 31. 1910.

11 Pötzl und Sch üller, Über die letale Hirnschwellung bei Syphilis. Zeitschr. f. d. ges. Neur. u. Psych. Orig. 3, 139.1910.

12. Nonne, Über Fälle von Symptomenkomplex „Tumor cerebri“ mit Ausgang in Heilung (Pseudotumor cerebri). Ưber letal verlaufende Fälle von Pseudotumor cerebri mit Sektionsbefund. Deutsche Zeitschr. f. Nervenheilk. $27,169.1904$.

13. - Über atypisch verlaufende Fälle von Hirnabsceß sowie weitere klinische und anatomische Beiträge zur Frage von Pseudotumor cerebri. Ebenda 33, 317. 1907.

14. Redlich, Über diffuse Hirnrindenveränderungen bei Hirntumoren. Festschr. f. Obersteiner 1, 320.1907.

15. Reichardt, Zur Entstehung des Hirndrucks bei Hirngeschwülsten und anderen Hirnkrankheiten und über eine bei diesen zu beobachtende besondere Art der Hirnschwellung. Deutsche Zeitschr. f. Nervenheilk. 28, 306. 1905.

16. - Über Todesfälle bei funktionellen Psychosen. Centralbl. f. Nervenheilk. 28, 1905.

17. - Über die Untersuchung des Gehirns mittels der Wage. Jena 1906.

18. - Ửber die Hirnschwellung. Zeitschr. f. d. ges. Neur. u. Psych. Ref. 3, 1. 1911 . 


\section{Erklärung der Tafel IX.}

Die sämtlichen Abbildungen sind mit homogener Immersion Zeiß 1/13, Komp. Ocular 4, bei Tubuslänge 160 gezeichnet.

Fig. 1. Pyramidenzellen der Rinde bei Fall II.

a) Alzheimers Methode $\mathrm{V}, \mathrm{b}-\mathrm{c}$ Thioninfärbung;

b) zeigt die vakuoläre Degeneration der Ganglienzelle;

c) dasselbe im leichten Grade, dabei sind die Protoplasmafortsätze angeschwollen; ngl. $=$ normale Gliazellen, pak $=$ präamöboide Gliakerne.

Fig. 2. Gliabeizegefrierschnitte aus dem Hemisphärenmark beim Fall II, Alzh. Meth. V. Homogenisation der Kerne in den protoplasmaarmen Gliazellen. (Entstehung der präamöboiden Kerne.)

Fig. 3. Gliabeizegefriersehnitte aus dem Balken im Fall II. Die lipoiden Einschlüsse osmiert in der Flemingschen Lösung, Färbung wie oben.

Amöboide Umwandlung der lipoidhaltigen faserbildenden Gliazellen: a-b) normale Elemente, c-e) beginnende Auflösung der Gliafasern, $\mathrm{f}-\mathrm{i}$ ) deutliche Homogenisation des Plasmas und Hyperchromatose des Kerns.

Fig. 4. Formolgefrierschnitte duroh die Wand des Seitenventrikels beim Fall I. Gegend der zwischen basalen Ganglien durchziehenden Faserzüge. Alz. heimers Meth. V. Große amöboide Gliazellen mit homogenem Plasmaleib und lipoiden Einschlüssen; uf = Übergangsform zwischen dem präamöboiden Kern und einer typischen amöboiden Gliazelle.

Fig. 5. Ventrikelwand beim Fall II. Tiefe Schichten des subependymalen Gliageflechtes, a-c) präamöboide Gliazellen.

d) beginnende Plasmaanhäufung um den Kern.

e-f) kleine homogene Formen der amöboiden Gliazelle.

g-i) große Elemente mit lipoiden Einsohlüssen und zahlreichen hydropischen Vakuolen. 